\title{
Neural mechanisms of cognitive reappraisal in remitted major depressive disorder
}

\author{
Moria J. Smoski ${ }^{1,{ }^{*}}$, Shian-Ling Keng ${ }^{2}$, Crystal Edler Schiller ${ }^{3}$, Jared Minkel ${ }^{3}$, and Gabriel S. \\ Dichter ${ }^{1,3,4}$ \\ ${ }^{1}$ Department of Psychiatry and Behavioral Sciences, Duke University Medical Center, Durham \\ NC 27710, USA \\ ${ }^{2}$ Department of Psychology and Neuroscience, Duke University, Durham NC 27708, USA \\ ${ }^{3}$ Department of Psychiatry, University of North Carolina at Chapel Hill School of Medicine, Chapel \\ Hill, NC 27599, USA \\ ${ }^{4}$ Duke-UNC Brain Imaging and Analysis Center, Duke University Medical Center, Durham, NC, \\ 27710, USA
}

\begin{abstract}
Background-Down-regulation of negative emotions by cognitive strategies relies on prefrontal cortical modulation of limbic brain regions, and impaired frontolimbic functioning during cognitive reappraisal has been observed in affective disorders. However, no study to date has examined cognitive reappraisal in unmedicated euthymic individuals with a history of major depressive disorder relative to symptom-matched controls. Given that a history of depression is a critical risk factor for future depressive episodes, investigating the neural mechanisms of emotion regulation in remitted major depressive disorder (rMDD) may yield novel insights into depression risk.
\end{abstract}

Method-We assessed 37 individuals (18 rMDD, 19 controls) with functional magnetic resonance imaging (fMRI) during a task requiring cognitive reappraisal of sad images.

Results-Both groups demonstrated decreased self-reported negative affect after cognitive reappraisal and no group differences in the effects of cognitive reappraisal on mood were evident. Functional MRI results indicated greater paracingulate gyrus (rostral anterior cingulate cortex, Brodmann area 32) activation and decreased right midfrontal gyrus (Brodmann area 6) activation during the reappraisal of sad images.

Limitations-Trial-by-trial ratings of pre-regulation affect were not collected, limiting the interpretation of post-regulation negative affect scores.

Conclusions-Results suggest that activation of rostral anterior cingulate cortex, a region linked to the prediction of antidepressant treatment response, and of the right midfrontal gyrus, a region involved in cognitive control in the context of cognitive reappraisal, may represent

(C) 2013 Elsevier B.V. All rights reserved.

Correspondence: Dr. Moria J. Smoski, Department of Psychiatry and Behavioral Sciences, Duke University Medical Center, Box 3026, Durham, NC 27710, USA, moria.smoski@duke.edu.

Publisher's Disclaimer: This is a PDF file of an unedited manuscript that has been accepted for publication. As a service to our customers we are providing this early version of the manuscript. The manuscript will undergo copyediting, typesetting, and review of the resulting proof before it is published in its final citable form. Please note that during the production process errors may be discovered which could affect the content, and all legal disclaimers that apply to the journal pertain. 
endophenotypic markers of future depression risk. Future prospective studies will be needed to validate the predictive utility of these neural markers.

\section{Keywords}

Emotion regulation; remitted major depression; prefrontal cortex; rostral anterior cingulate cortex; fMRI

\section{Introduction}

Effective emotion regulation is a critical factor for psychological health, and functional magnetic resonance imaging (fMRI) has implicated a number of frontolimbic brain regions in emotion regulation (Ochsner et al., 2004). In adults without psychopathology, prefrontal cortical regions, including dorsolateral prefrontal cortex, ventrolateral prefrontal cortex, and anterior cingulate cortex modulate emotional responses in limbic regions (Ochsner and Gross, 2008). Within this regulatory circuit, the medial prefrontal cortex and rostral anterior cingulate cortex are positively associated with activation in prefrontal cognitive control regions and negatively associated with limbic activation (Urry et al., 2006; Siegle et al., 2006).

Major depressive disorder (MDD) is characterized by dysregulated biological, cognitive, and behavioral responses during affect processing (Davidson et al., 2002; Ressler and Mayberg, 2007). Additionally, a handful of studies have documented differential activation of frontolimbic brain regions during emotion regulation in MDD. Johnstone and collegues (2007) reported an inverse relation between left ventral medial prefrontal cortex activation and amygdala activation in controls but the opposite pattern in outpatients with MDD during cognitive reappraisal, a form of emotion regulation in which the meaning of an emotional stimulus is reinterpreted to change its affective tone. Consistent with this finding, Greening and colleagues (2013) reported differential prefrontal cortical influence on amygdala activation in MDD during regulation of both negative and positive emotions. Similarly, Beauregard and colleagues (2006) reported hyperactivation in dorsal anterior cingulate cortex, right amygdala, and right insula in MDD during down-regulation of responses to sad film clips, and Erk and colleagues (2010) reported reduced prefrontal activation, reduced prefrontolimbic coupling, and decreased capacity to reduce amygdala activation to negative pictures in a manner that predicted depressive symptom severity. However, Dillon \& Pizzagalli (2013) reported evidence of successful modulation of brain activation in MDD during reappraisal of negative stimuli in the context of specific instructions to dampen emotion responses by increasing the sense of psychological distance, suggesting constraints on the effects of MDD on the neural correlates of cognitive reappraisal.

Despite evidence of altered neural responses in MDD during emotion regulation, it is unclear whether currently euthymic individuals with remitted MDD (rMDD) show similar patterns of frontolimbic dysregulation, a finding that would suggest a marker of MDD vulnerability, or, conversely, whether brain-based changes in regulation are constrained to active depressive symptom states. Major depressive disorder is cyclical, with previous depressive episodes serving as a powerful risk factor for future episodes (Lewinsohn et al., 1988). Cognitive vulnerability theories of MDD posit that individuals at heightened MDD risk are characterized by negative cognitive biases, including negative self-evaluations (Gemar et al., 2001) and attentional bias towards negative stimuli (De Raedt and Koster, 2010). In euthymic individuals with a history of MDD, these biases may be evident only in the context of an affective stressor (Scher et al., 2005), highlighting the critical importance of effective emotion regulation in individuals with a history of MDD to protect against future depressive episodes. Given that rMDD is associated with heightened vulnerability to 
future MDD episodes, identifying endophenotypes related to rMDD status may suggest novel preventative intervention targets.

Euthymic individuals with a history of MDD show a range of altered neurocognitive and neurobiological profiles, including impairments in cognitive control (Vanderhasselt and De Raedt, 2009), altered resting regional homogeneity in frontal, temporal and parietal lobes (Yuan et al., 2008), and deficits in attention and executive functions (Paelecke-Habermann et al., 2005). Euthymic individuals with a history of MDD also show increased orbitofrontal and anterior cingulate cortex activity in response to sad images, suggestive of MDD-like emotion regulation deficits (Liotti et al., 2002). In addition, rMDD is characterized by greater left dorsolateral prefrontal cortex activity to negative emotional distracters during working memory (Kerestes et al., 2011) and higher calcarine cortex activity to sad film clips (Farb et al., 2011), potentially reflecting compensatory activation to maintain adequate task performance in the context of negative affective cues.

The purpose of the present study was to examine the neural mechanisms of cognitive reappraisal of sad images in unmedicated individuals with a history of MDD. Cognitive reappraisal is an effective emotion regulation strategy (Gross, 2002) that predicts depressive symptom severity (Kraaij et al., 2002; Gross and John, 2003; Joormann and Gotlib, 2010) and that may convey protection against the impact of stressful events (Sears et al., 2003). We are aware of one previous fMRI study of emotion regulation in rMDD: Kanske and colleagues (2012) examined response to negative images and reported increased amygdala and orbitofrontal activation during cognitive reappraisal and increased amygdala and anterior cingulate cortex activation during distraction in rMDD. However, a significant proportion of the rMDD group was taking psychiatric medications, and depressive symptom severity differed between the rMDD and control groups, confounding the effects of MDD history with the effects of current MDD symptoms. The present study builds on the findings of Kanske and colleagues (2012) by examining brain activation patterns during cognitive reappraisal in an unmedicated rMDD sample compared to sample of controls matched on (low) current MDD symptom severity. Given that rMDD is characterized by deficits in cognitive control (Vanderhasselt and De Raedt, 2009), particularly in affective contexts (De Raedt and Koster, 2010), we predicted compensatory increases in prefrontal cortical activation during cognitive reappraisal, akin to the findings of Kanske et al (2012), and that activation of prefrontal cortical regions linked to emotion regulation would be associated with levels of negative affect following reappraisal.

\section{Methods}

\subsection{Participants}

Nineteen affectively healthy right-handed adult control participants ( 7 male; 15 Caucasian; $27.9 \pm 6.3$ years old; all right-handed) were recruited from lists of potential participants maintained by the Duke-UNC Brain Imaging and Analysis Center (BIAC). Nineteen adults with rMDD (4 male; 13 Caucasian; $24.5 \pm 5.4$ years old; 17 right-handed) were recruited via a participant database maintained at the Cognitive Behavioral Research and Treatment Program at Duke University Medical Center. Data from one rMDD participant were excluded due to an elevated Beck Depression Inventory-II (BDI; Beck et al., 1996) score on the day of the scan $(\mathrm{BDI}=30)$, resulting in a final sample of 18 rMDD participants. Exclusion criteria for both groups included age $<19$ or $>55$ years old, current Axis I psychopathology, psychiatric medication use within the past month, verbal IQ scores (estimated by the North American Adult Reading Test $(U t t l, 2002)$ ) < 80, BDI > 8, or MRI contraindications. Inclusion in the rMDD group was contingent on a prior diagnosis of MDD based on SCID I semi-structured interview (First et al., 1996). Control participants were lifetime-free of MDD. None of the control participants and two rMDD participants 
were receiving psychotherapy at the time of participation. Five rMDD participants had previously used psychotropic medications. All participants consented to a protocol approved by the local Human Investigations Committees at both UNC-Chapel Hill and Duke University Medical Centers and were paid $\$ 35$ for completing the imaging portion of the study. All participants had normal or corrected-to-normal vision and completed a mock scan session prior to imaging. Information about demographics and prior MDD episodes are presented in Table 1.

\section{2. fMRI task}

Each trial began with a fixation cross $(6 \mathrm{~s})$ followed by presentation of a sad or neutral picture (Figure 1 depicts the timing and content of the task). After initial picture display (6 to $9 \mathrm{~s}$, jittered) a visual regulation instruction was superimposed on the bottom of the picture, indicating the regulation strategy to use. Regulation continued for $5 \mathrm{~s}$ following image offset. Finally, participants rated their post-trial affect using a visual analogue scale. The task included three regulation conditions. In the "attend" condition, used with both sad and neutral pictures, participants were instructed not to regulate their emotion response. In the "reappraise" condition, used with only sad images, participants were asked to reinterpret the image to reduce its negative tone. Both self-focused and situation-focused reappraisal strategies were permitted (Ochsner et al., 2004). Results from a third condition, "accept," are not reported here. Four runs of twelve trials each were administered (48 trials total; 4'24" per run), and there were 12 trials for each regulation condition.

Immediately prior to the scan, participants learned and practiced the regulation strategies with an experimenter until they could correctly implement them without assistance. Task images were drawn from two sources: (1) sad images from the International Affective Picture System based on normative sadness ratings (Mikels et al., 2005), and (2) a normed set of sad and neutral images used in previous MDD imaging studies (Wang et al., 2005; Wang et al., 2008; Dichter et al., 2009; Dichter et al., 2010).

\subsection{Imaging methods}

Scanning was performed on a General Electric (Waukesha, Wisconsin, USA) MR750 3.0 Tesla scanner equipped with high-power high-duty-cycle $50-\mathrm{mT} / \mathrm{m}$ gradients at $200 \mathrm{~T} / \mathrm{m} / \mathrm{s}$ slew rate and a 32-channel head coil for parallel imaging. A quadrature birdcage radio frequency head coil was used for transmit and receive. A high resolution T1-weighted image with 166 slices was acquired using a 3D FSPGR pulse sequence $(\mathrm{TR}=7.484 \mathrm{~ms}$; $\mathrm{TE}=$ $2.984 \mathrm{~ms} ; \mathrm{FOV}=256 \mathrm{~mm}$; image matrix $=256 \times 256$; voxel size $=1 \mathrm{~mm}^{3}$ ) and used for coregistration with the functional data. This structural image was aligned in a near axial plane defined by the anterior and posterior commissures. Whole brain functional images were acquired using a spiral pulse sequence with SENSE reconstruction sensitive to blood oxygenation level dependent contrast (TR, $1500 \mathrm{~ms}$; TE, $30 \mathrm{~ms}$; FOV, $256 \mathrm{~mm}$; image matrix, $64 \times 64 ; \alpha=60^{\circ}$; voxel size $=4 \mathrm{~mm}^{3} ; 32$ axial slices). Functional images were aligned similarly to the T1-weighted structural image. A semi-automated high-order shimming program ensured global field homogeneity.

\subsection{Imaging data analysis}

Functional data were preprocessed using FSL version 4.1.8 (Oxford Centre for Functional Magnetic Resonance Imaging of the Brain (FMRIB), Oxford University, U.K.). Preprocessing was applied in the following steps: (i) brain extraction for non-brain removal (Smith et al., 2004), (ii) motion correction using MCFLIRT (Smith, 2002), (iii) spatial smoothing using a Gaussian kernel of FWHM $5 \mathrm{~mm}$, (iv) mean-based intensity normalization of all volumes by the same factor, and (v) high-pass filtering (Jenkinson et al., 2002). Functional images of each participant were co-registered to structural images in 
native space, and structural images were normalized into a standard stereotaxic space (Montreal Neurological Institute) for intersubject comparison. This transformation included resampling voxel sizes to $2 \mathrm{~mm}^{3}$. The same transformation matrices used for structural-tostandard transformations were then used for functional-to-standard space transformations of co-registered functional images. All registrations were carried out using an intermodal registration tool (Jenkinson et al., 2002; Smith et al., 2004). Voxel-wise temporal autocorrelation was estimated and corrected using FMRIB's Improved Linear Model (FILM; Jenkinson and Smith, 2001). Onset times of events were used to model a signal response containing a regressor for each strategy, which was convolved with a double- $\gamma$ function to model the hemodynamic response. Model fitting generated whole brain images of parameter estimates and variances representing average signal change from baseline. Group-wise activation images were calculated by a mixed effects higher level analysis using Bayesian estimation techniques, FMRIB Local Analysis of Mixed Effects (FILM, Woolrich et al., 2001; Smith et al., 2004).

An a priori mask was created for small volume correction that included the frontal lobes and bilateral amygdala generated in FSL using the Harvard-Oxford cortical and subcortical structural probabilistic atlases. Masks were thresholded at 25\%, binarized, and then combined into a single mask using fslmaths. For all analyses, voxels were considered significant if they passed a statistical threshold of $p<.005$, uncorrected, and were part of a 35 -voxel $\left(280 \mathrm{~mm}^{3}\right)$ cluster of contiguous significant voxels, resulting in a cluster-corrected significance threshold of $p<.05$. This cluster size was determined by performing 1000 Monte Carlo simulations using 3dClustSim (Ward, 2000).

Pre-regulation and regulation phases of the task were analyzed separately. General linear models evaluated clusters that showed significant interactions of Group (rMDD, control) with trial type (sad relative to neutral images for the pre-regulation phase; reappraise-sad relative to attend-sad for the regulation phase). Activation localizations were based on Harvard-Oxford cortical and subcortical structural probabilistic atlases, with Brodmann area identification via the Talairach Daemon, as implemented in FSL View v3.1.8. Exploratory correlation analyses between brain activation and clinical characteristics of the rMDD group were conducted by extracting contrast estimates from each participant and condition within significant clusters identified by the whole-brain general linear models described above.

\section{Results}

\section{Emotion regulation self-report}

In-scanner self-reported emotion regulation was evaluated via a 2 (Group: rMDD, control) $\times$ 3 (Trial Type: attend-sad, attend-neutral, reappraise-sad) repeated measures ANOVA. There was a significant main effect of Trial Type, $F(2,70)=103.67, p<.0001$. Follow-up paired $t$ tests indicated more negative affect ratings on attend-sad trials than reappraise-sad trials, $t(36)=5.06, p<.001$, and more negative affect ratings on reappraise-sad trials than attendneutral trials, $t(36)=8.20, p<.001$. There was no main effect of Group, $F(1,35)=0.96, p=$. 34 , or Group $\times$ Trial Type interaction, $F(2,70)=1.82, p=.17$. These results indicate that both groups reported successful emotion regulation, with no differences between groups.

\section{Imaging Data: Group Differences during Pre-Regulation}

Though not a central focus of this investigation, the pre-regulation phase was analyzed via a Group (rMDD, control) $\times$ Trial Type (sad images, neutral images) model. This model revealed that the rMDD group was characterized by relatively decreased activation in left frontal pole, bilateral inferior frontal gyrus, right middle frontal gyrus, and left precentral gyrus during the pre-regulation condition (see Table 2). Conversely, there were no clusters 
with greater activation in the rMDD group. To evaluate the influence of left-handedness on these results, the same model was evaluated omitting the two left-handed participants, and significant $\mathrm{rMDD}<\mathrm{control}$ clusters remained in these regions. To evaluate the influence of current psychotherapy use on these results, the same model was evaluated omitting the two participants who were receiving psychotherapy. The left inferior frontal gyrus cluster was no longer significant, but the rMDD<control significant clusters in the other regions remained.

\section{Imaging Data: Group Differences during Emotion Regulation}

Of central interest were group differences during emotion regulation. Group (rMDD, control) $\times$ Trial Type (reappraise-sad, attend-sad) models during emotion regulation (see Table 3 and Figure 2) revealed that the rMDD group was characterized by relatively decreased activation in right middle frontal gyrus (Brodmann area 6) and by relatively greater activation in right paracingulate gyrus (Brodmann's area 32 within rostral anterior cingulate cortex (rACC)). Group- and condition-averaged signal intensities extracted from these clusters revealed that, for the right middle frontal gyrus cluster, the rMDD demonstrated significantly decreased activation relative to the control group during the reappraisal of sad images $(p<.05)$, and the control group demonstrated greater activation during reappraisal of sad images relative to attending to neural images $(p<.001)$. For the rACC cluster, the rMDD group showed greater activation during the reappraisal of sad images relative to attending to sad images $(p<.003)$ and a trend towards decreased activation relative to the control group during the attend condition $(p<.06)$. To evaluate the influence of left-handedness and current psychotherapy use on these results, the same model was evaluated first omitting the two left-handed participants and next omitting the two participants who were receiving psychotherapy, and these clusters remained significant.

\section{Correlations between brain activation and self-reported emotion regulation in the rMDD group}

To test for relations between brain activation magnitudes and self-reported emotion regulation in the rMDD group, correlations between activation magnitudes within clusters reported in Tables 2 and 3 and in-scanner reports of post-reappraisal affect were evaluated. Even at uncorrected significance thresholds, no relations were significant.

\section{Discussion}

The purpose of this study was to extend the literature on the neural correlates of emotion regulation in MDD to currently euthymic individuals with a history of MDD. Studying individuals with a prior history of depressive episodes may clarify mechanisms by which MDD history conveys vulnerability to future depressive episodes, and the elucidation of endophenotypes linked to MDD in patients with a history of the disorder is a necessary, though not sufficient, criteria contributing to the identification of a risk marker for a disorder (Alloy et al., 1999).

Given the growing literature documenting differential prefrontal cortex and amygdala activations during emotion regulation in MDD, (Johnstone et al., 2007; Greening et al., 2013) (Beauregard et al., 2006; Erk et al., 2010), primary analyses focused on the contrast of cognitive reappraisal of sad images versus attending to sad images. We predicted that the rMDD group would show relatively increased activation in prefrontal cortical regions associated with cognitive control in the context of affective processing (Ochsner et al., 2002; Ochsner et al., 2004). Results partially confirmed hypotheses, as we found evidence of relatively greater activation in $\mathrm{rACC}$, though we also found relatively decreased activation in right middle frontal gyrus. 
The middle frontal gyrus and the rACC play critical functional roles in MDD. The middle frontal gyrus is recruited during tasks involving working memory, selective attention, and successful emotion regulation (Ochsner and Gross, 2008; Ochsner et al., 2004). Multiple studies have documented decreased middle frontal gyrus activity in MDD during tasks involving emotion processing (Feeser et al., 2013; Dichter et al., 2009), cognitive control (Kikuchi et al., 2012; Okada et al., 2009), and, most relevant in the present context, emotion regulation (Wang et al., 2008). Additionally, reduced pre-treatment middle frontal gyrus activity is a predictor of worse antidepressant treatment response (Samson et al., 2011; Lisiecka et al., 2011). We thus interpret the present findings of relatively decreased middle frontal gyrus activity in rMDD during emotion regulation of sad images to potentially reflect decreased neural resources to exert cognitive control, despite the fact that perhaps relatively low task demands were not sufficient to yield group differences in self-reported emotion regulation success.

The rACC, on the other hand, has emerged as a promising predictor of antidepressant treatment response (Mayberg, 1997; Mayberg et al., 1997; Saxena et al., 2003; Kennedy et al., 2007). A recent comprehensive meta-analysis of 23 studies that considered functional neuroimaging predictors of a range of antidepressant treatments, including pharmacology, electroconvulsive therapy, rapid transcranial magnetic stimulation, sleep deprivation, and psychotherapy, as well as a range of imaging modalities, including electroencephalography, magnetoencephalography, fMRI, positron emission tomography, and single photon emission computed tomography reported that increased rACC activity is a robust predictor of improved treatment response across various treatments and imaging modalities (Pizzagalli, 2011). One theory for the linkage between rACC activity and antidepressant treatment response is its location as a "hub" position within the default mode network (Shackman et al., 2011) that mediates adaptive self-referential processing as well as task-switching with dorsal cognitive control networks in depression (Pizzagalli, 2011). In this regard, the well documented biased emotion processing that characterizes depression (Gotlib and Joormann, 2010) may be rooted in increased tonic rACC activation that interferes with task-appropriate activation. In this regard, increased rACC activation may be a marker of improved emotional functioning in individuals who recovered from a recent episode of depression.

In-scanner self-reported mood after emotion regulation revealed that both the rMDD and control groups were successful at down-regulating their responses to sad images, and groups did not differ in these ratings, consistent with prior reports (Greening et al., 2013; Dillon and Pizzagalli, 2013). The fact that group differences in fMRI were observed in the absence of divergence in self-reported affect further supports the compensatory framework outlined above with respect to rACC activation.

The present finding of increased prefrontal cortex activation during cognitive reappraisal partially extends the results of Kanske and colleagues (2012) who reported relatively increased right orbitofrontal cortex activation during cognitive reappraisal and relatively increased dorsomedial prefrontal cortex activation during distraction regulation in rMDD. Kanske et al. (2012) used high-arousal negative images, including mutilation images, whereas the present study used images selected specifically for sad (i.e., low arousal) content. Sad stimuli may be more personally relevant to individuals with a history of MDD than stimuli eliciting physical threat or disgust (Mathews et al., 1996), which may in turn promote more self-relevant cognitions (Wood et al., 1990). In this regard, the particular stimulus sets used here may have contributed to localization of hyperactive responses in rMDD in the present study to the rACC (Northoff et al., 2006).

Of note, whereas we did not find group differences in amygdala activation during cognitive reappraisal, Kanske et al. (2012) reported decreased down-regulation of amygdala responses 
in rMDD during cognitive reappraisal. This may be related to the disparity in depressive symptom severity in the rMDD groups in both studies. In the present study, groups were matched on current depressive symptom severity, whereas rMDD participants had significantly more depressive symptoms than controls in Kanske et al. (2012). Thus, aberrant prefrontal cortex activation during emotion regulation may characterize rMDD in the context of a range of subclinical depressive symptoms, whereas differences in prefrontal modulatory effects on amygdala may not be evident in rMDD participants without significant depressive symptoms.

Additionally, decreased middle frontal gyrus activity in rMDD was not reported in Kanske et al. (2012). Reappraisal requires a reinterpretation of the meaning of an emotional stimulus to change its affective tone, but the form of that reinterpretation varies across individuals. For example, self-focused strategies, which include taking a detached, third-party perspective on a negative stimulus, and situation-focused strategies, which involve making a positive or optimistic reinterpretation of a negative stimulus, have been found to activate overlapping but differentiable networks. The current study permitted both self-focused and situation-focused reappraisal, and relatively decreased activation in right middle frontal gyrus in rMDD is consistent with activation implicated in situation-focused reappraisal (Ochsner et al., 2004).

Although group differences during the pre-regulatory phase of the task was not a primary focus of the present study, during pre-regulation we found relatively less activation in a number of prefrontal cortical regions previously shown to activate during emotion induction (Steele and Lawrie, 2004; Kober et al., 2008) and regulation (Ochsner et al., 2004). Thus, activation of these regions during pre-regulation may reflect automatic, nonconscious affective regulatory processes activated to a lesser degree in the rMDD group while viewing sad images.

One limitation in our design is the lack of measurement of trial-by-trial pre-regulation affect, so group differences in subjective regulation success could not be directly measured. However, the lack of group differences in self-reported negative affect to attend-sad trials suggests that the groups did not differ in emotional reactivity to the sad images. Across groups, lower negative affect scores following reappraisal versus attend-sad trials suggest that reappraisal was associated with successful regulation. Interpretation of the clinical relevance of the findings would be improved with data on long-term clinical course, to test if neural activation during cognitive reappraisal predicts continued remission versus relapse.

In summary, in the absence of group differences in self-reported emotion regulation effectiveness, we found that currently euthymic individuals with a history of MDD showed decreased right middle frontal gyrus activation and increased rACC activation during cognitive reappraisal of sad images. These findings were evident despite the fact that rMDD and controls groups were matched to have equivalently low levels of depressive symptom severity. These patterns of brain activity suggest a possible endophenotypic marker of risk for future depression given that a prior history of depressive episodes represents a powerful risk factor for future episodes (Lewinsohn et al., 1988). Further research is needed to determine whether these patterns of brain activation prospectively convey greater risk for future MDD episodes or are present before the onset of a first depressive episode.

\section{Acknowledgments}

The authors thank Josh Bizzell and Chris Petty for assistance with image analysis, Alison Rittenberg for assistance with data collection, and MRI technologists Susan Music, Natalie Goutkin, and Luke Poole for assistance with data acquisition. 
Assistance for this study was provided by the Neuroimaging Core of the Carolina Institute for Developmental Disabilities (P30 HD03110). This research was supported by grants from the NARSAD Young Investigator Program. Investigator effort was supported by NIMH K23 MH087754 to M. Smoski and NIMH K23 MH081285 to G. Dichter.

\section{REFERENCE}

Alloy, LB.; Ambramson, LY.; Raniere, D.; Dyller, IM. Research methods in adult psychopathology. In: Kendall, PC.; Butcher, JN.; Holmbeck, GN., editors. Handbook of research methods in clinical psychology. 2nd ed.. New York: Wiley; 1999.

Beauregard M, Paquette V, Levesque J. Dysfunction in the neural circuitry of emotional selfregulation in major depressive disorder. Neuroreport. 2006; 17:843-846. [PubMed: 16708026]

Beck, AT.; Steer, RA.; Brown, GK. Manual for Beck Depression Inventory-II. San Antonio, TX: Psychological Corporation; 1996.

Davidson RJ, Pizzagalli D, Nitschke JB, Putnam K. Depression: perspectives from affective neuroscience. Annu Rev Psychol. 2002; 53:545-574. [PubMed: 11752496]

De Raedt R, Koster EH. Understanding vulnerability for depression from a cognitive neuroscience perspective: A reappraisal of attentional factors and a new conceptual framework. Cogn Affect Behav Neurosci. 2010; 10:50-70. [PubMed: 20233955]

Dichter GS, Felder JN, Smoski MJ. Affective context interferes with cognitive control in unipolar depression: an fMRI investigation. Journal of Affective Disorders. 2009; 114:131-142. [PubMed: 18706701]

Dichter GS, Felder JN, Smoski MJ. The effects of Brief Behavioral Activation Therapy for Depression on cognitive control in affective contexts: An fMRI investigation. Journal of Affective Disorders. 2010; 126:236-244. [PubMed: 20421135]

Dillon DG, Pizzagalli DA. Evidence of successful modulation of brain activation and subjective experience during reappraisal of negative emotion in unmedicated depression. Psychiatry Res. 2013; 212:99-107. [PubMed: 23570916]

9. Erk S, Mikschl A, Stier S, Ciaramidaro A, Gapp V, Weber B, Walter H. Acute and sustained effects of cognitive emotion regulation in major depression. J Neurosci. 2010; 30:15726-15734. [PubMed: 21106812]

Farb NA, Anderson AK, Bloch RT, Segal ZV. Mood-Linked Responses in Medial Prefrontal Cortex Predict Relapse in Patients with Recurrent Unipolar Depression. Biol Psychiatry. 2011

Feeser M, Schlagenhauf F, Sterzer P, Park S, Stoy M, Gutwinski S, Dalanay U, Kienast T, Bauer M, Heinz A, Strohle A, Bermpohl F. Context insensitivity during positive and negative emotional expectancy in depression assessed with functional magnetic resonance imaging. Psychiatry Res. 2013; 212:28-35. [PubMed: 23473989]

First, MB.; Spitzer, RL.; Gibbon, M.; Williams, JBW. Structured Clinical Interview for DSM-IV Axis I Disorders (SCID), Clinician Version; Administration Booklet. Washington, D.C: American Psychiatric Press; 1996.

Gemar MC, Segal ZV, Sagrati S, Kennedy SJ. Mood-induced changes on the Implicit Association Test in recovered depressed patients. Journal of Abnormal Psychology. 2001; 110:282-289. [PubMed: 11358022]

Gotlib IH, Joormann J. Cognition and depression: current status and future directions. Annu Rev Clin Psychol. 2010; 6:285-312. [PubMed: 20192795]

Greening SG, Osuch EA, Williamson PC, Mitchell DG. The neural correlates of regulating positive and negative emotions in medication-free major depression. Soc Cogn Affect Neurosci. 2013

Gross JJ. Emotion regulation: Affective, cognitive, and social consequences. Psychophysiology. 2002; 39:281-291. [PubMed: 12212647]

Gross JJ, John OP. Individual differences in two emotion regulation processes: implications for affect, relationships, and well-being. Journal of Personality \& Social Psychology. 2003; 85:348-362. [PubMed: 12916575]

Jenkinson M, Bannister P, Brady M, Smith S. Improved optimization for the robust and accurate linear registration and motion correction of brain images. Neuroimage. 2002; 17:825-841. [PubMed: 12377157] 
Jenkinson M, Smith S. A global optimisation method for robust affine registration of brain images. Med Image Anal. 2001; 5:143-156. [PubMed: 11516708]

Johnstone T, Van Reekum CM, Urry HL, Kalin NH, Davidson RJ. Failure to regulate: counterproductive recruitment of top-down prefrontal-subcortical circuitry in major depression. $\mathrm{J}$ Neurosci. 2007; 27:8877-8884. [PubMed: 17699669]

Joormann J, Gotlib IH. Emotion regulation in depression: Relation to cognitive inhibition. Cognition \& Emotion. 2010; 24:281-298. [PubMed: 20300538]

Kanske P, Heissler J, Schönfelder S, Wessa M. Neural correlates of emotion regulation deficits in remitted depression: The influence of regulation strategy, habitual regulation use emotional valence. NeuroImage. 2012; 61:686-693. [PubMed: 22613776]

Kennedy SH, Konarski JZ, Segal ZV, Lau MA, Bieling PJ, Mcintyre RS, Mayberg HS. Differences in brain glucose metabolism between responders to CBT and venlafaxine in a 16-week randomized controlled trial. Am J Psychiatry. 2007; 164:778-788. [PubMed: 17475737]

Kerestes R, Ladouceur CD, Meda S, Nathan PJ, Blumberg HP, Maloney K, Ruf B, Saricicek A, Pearlson GD, Bhagwagar Z, Phillips ML. Abnormal prefrontal activity subserving attentional control of emotion in remitted depressed patients during a working memory task with emotional distracters. Psychol Med. 2011:1-12.

Kikuchi T, Miller JM, Schneck N, Oquendo MA, Mann JJ, Parsey RV, Keilp JG. Neural responses to incongruency in a blocked-trial Stroop fMRI task in major depressive disorder. J Affect Disord. 2012; 143:241-247. [PubMed: 22995943]

Kober H, Barrett LF, Joseph J, Bliss-Moreau E, Lindquist K, Wager TD. Functional grouping and corticalâ€"subcortical interactions in emotion: A meta-analysis of neuroimaging studies. NeuroImage. 2008; 42:998-1031. [PubMed: 18579414]

Kraaij V, Pruymboom E, Garnefski N. Cognitive coping and depressive symptoms in the elderly: a longitudinal study. Aging \& Mental Health. 2002; 6:275-281. [PubMed: 12217096]

Lewinsohn PM, Hoberman HM, Rosenbaum M. A prospective study of risk factors for unipolar depression. J Abnorm Psychol. 1988; 97:251-264. [PubMed: 3192816]

Liotti M, Mayberg HS, Mcginnis S, Brannan SL, Jerabek P. Unmasking disease-specific cerebral blood flow abnormalities: mood challenge in patients with remitted unipolar depression. Am J Psychiatry. 2002; 159:1830-1840. [PubMed: 12411216]

Lisiecka D, Meisenzahl E, Scheuerecker J, Schoepf V, Whitty P, Chaney A, Moeller HJ, Wiesmann M, Frodl T. Neural correlates of treatment outcome in major depression. Int $\mathbf{J}$ Neuropsychopharmacol. 2011; 14:521-534. [PubMed: 21205435]

Mathews A, Ridgeway V, Williamson DA. Evidence for attention to threatening stimuli in depression. Behaviour Research and Therapy. 1996; 34:695-705. [PubMed: 8936752]

Mayberg HS. Limbic-cortical dysregulation: a proposed model of depression. J Neuropsychiatry Clin Neurosci. 1997; 9:471-481. [PubMed: 9276848]

Mayberg HS, Brannan SK, Mahurin RK, Jerabek PA, Brickman JS, Tekell JL, Silva JA, Mcginnis S, Glass TG, Martin CC, Fox PT. Cingulate function in depression: a potential predictor of treatment response. Neuroreport. 1997; 8:1057-1061. [PubMed: 9141092]

Mikels JA, Fredrickson BL, Larkin GR, Lindberg CM, Maglio SJ, Reuter-Lorenz PA. Emotional category data on images from the International Affective Picture System. Behavior Research Methods. 2005; 37:626-630. [PubMed: 16629294]

Northoff G, Heinzel A, De Greck M, Bermpohl F, Dobrowolny H, Panksepp J. Self-referential processing in our brain -- A meta-analysis of imaging studies on the self. NeuroImage. 2006; 31:440-457. [PubMed: 16466680]

Ochsner KN, Bunge SA, Gross JJ, Gabrieli JD. Rethinking feelings: An f MRI study of the cognitive regulation of emotion. Journal of Cognitive Neuroscience. 2002; 14:1215-1229. [PubMed: 12495527]

Ochsner KN, Gross JJ. Cognitive emotion regulation: Insights from social cognitive and affective neuroscience. Current Directions in Psychological Science. 2008; 17:153-158.

Ochsner KN, Ray RD, Cooper JC, Robertson ER, Chopra S, Gabrieli JDE, et al. For better or for worse: Neural systems supporting the cognitive down- and up-regulation of negative emotion. Neuroimage. 2004; 23:483-499. [PubMed: 15488398] 
Okada G, Okamoto Y, Yamashita H, Ueda K, Takami H, Yamawaki S. Attenuated prefrontal activation during a verbal fluency task in remitted major depression. Psychiatry Clin Neurosci. 2009; 63:423-425. [PubMed: 19566776]

Paelecke-Habermann Y, Pohl J, Leplow B. Attention and executive functions in remitted major depression patients. J Affect Disord. 2005; 89:125-135. [PubMed: 16324752]

Pizzagalli DA. Frontocingulate dysfunction in depression: toward biomarkers of treatment response. Neuropsychopharmacology. 2011; 36:183-206. [PubMed: 20861828]

Ressler KJ, Mayberg HS. Targeting abnormal neural circuits in mood and anxiety disorders: from the laboratory to the clinic. Nat Neurosci. 2007; 10:1116-1124. [PubMed: 17726478]

Samson AC, Meisenzahl E, Scheuerecker J, Rose E, Schoepf V, Wiesmann M, Frodl T. Brain activation predicts treatment improvement in patients with major depressive disorder. J Psychiatr Res. 2011; 45:1214-1222. [PubMed: 21477817]

Saxena S, Brody AL, Ho ML, Zohrabi N, Maidment KM, Baxter LR Jr. Differential brain metabolic predictors of response to paroxetine in obsessive-compulsive disorder versus major depression. Am J Psychiatry. 2003; 160:522-532. [PubMed: 12611834]

Scher CD, Ingram RE, Segal ZV. Cognitive reactivity and vulnerability: Empirical evaluation of construct activation and cognitive diatheses in unipolar depression. Clinical Psychology Review. 2005; 25:487-510. [PubMed: 15914266]

Sears SR, Stanton AL, Danoff-Burg S. The yellow brick road and the emerald city: Benefit finding, positive reappraisal coping and posttraumatic growth in women with early-stage breast cancer. Health Psychology. 2003; 22:487-497. [PubMed: 14570532]

Shackman AJ, Salomons TV, Slagter HA, Fox AS, Winter JJ, Davidson RJ. The integration of negative affect, pain and cognitive control in the cingulate cortex. Nat Rev Neurosci. 2011; 12:154-167. [PubMed: 21331082]

Siegle GJ, Carter CS, Thase ME. Use of FMRI to predict recovery from unipolar depression with cognitive behavior therapy. Am J Psychiatry. 2006; 163:735-738. [PubMed: 16585452]

Smith SM. Fast robust automated brain extraction. Human Brain Mapping. 2002; 17:143-155. [PubMed: 12391568]

Smith SM, Jenkinson M, Woolrich MW, Beckmann CF, Behrens TEJ, Johansen-Berg H, Bannister PR, De Luca M, Drobnjak I, Flitney DE, Niazy RK, Saunders J, Vickers J, Zhang Y, De Stefano N, Brady JM, Matthews PM. Advances in functional and structural MR image analysis and implementation as FSL. Neuroimage. 2004; 23:S208-S219. [PubMed: 15501092]

Steele JD, Lawrie SM. Segregation of cognitive and emotional function in the prefrontal cortex: a stereotactic meta-analysis. NeuroImage. 2004; 21:868-875. [PubMed: 15006653]

Urry HL, Van Reekum CM, Johnstone T, Kalin NH, Thurow ME, Schaefer HS, Jackson CA, Frye CJ, Greischar LL, Alexander AL, Davidson RJ. Amygdala and ventromedial prefrontal cortex are inversely coupled during regulation of negative affect and predict the diurnal pattern of Cortisol secretion among older adults. J Neurosci. 2006; 26:4415-4425. [PubMed: 16624961]

Uttl B. North American Adult Reading Test: age norms, reliability, and validity. J Clin Exp Neuropsychol. 2002; 24:1123-1137. [PubMed: 12650237]

Vanderhasselt MA, De Raedt R. Impairments in cognitive control persist during remission from depression and are related to the number of past episodes: An event related potentials study. Biological Psychology. 2009; 81:169-176. [PubMed: 19482234]

Wang L, Labar KS, Smoski M, Rosenthal MZ, Dolcos F, Lynch TR, Krishnan RR, Mccarthy G. Prefrontal mechanisms for executive control over emotional distraction are altered in major depression. Psychiatry Res. 2008; 163:143-155. [PubMed: 18455373]

Wang L, Mccarthy G, Song AW, Labar KS. Amygdala activation to sad pictures during high-field (4 Tesla) functional magnetic resonance imaging. Emotion. 2005; 5:12-22. [PubMed: 15755216]

Wood JV, Saltzberg JA, Goldsamt LA. Does affect induce self-focused attention? Journal of Personality and Social Psychology. 1990; 58:899-908. [PubMed: 2348375]

Woolrich MW, Ripley BD, Brady M, Smith SM. Temporal autocorrelation in univariate linear modeling of FMRI data. Neuroimage. 2001; 14:1370-1386. [PubMed: 11707093] 
Yuan Y, Zhang Z, Bai F, Yu H, Shi Y, Qian Y, Liu W, You J, Zhang X, Liu Z. Abnormal neural activity in the patients with remitted geriatric depression: a resting-state functional magnetic resonance imaging study. J Affect Disord. 2008; 111:145-152. [PubMed: 18372048] 


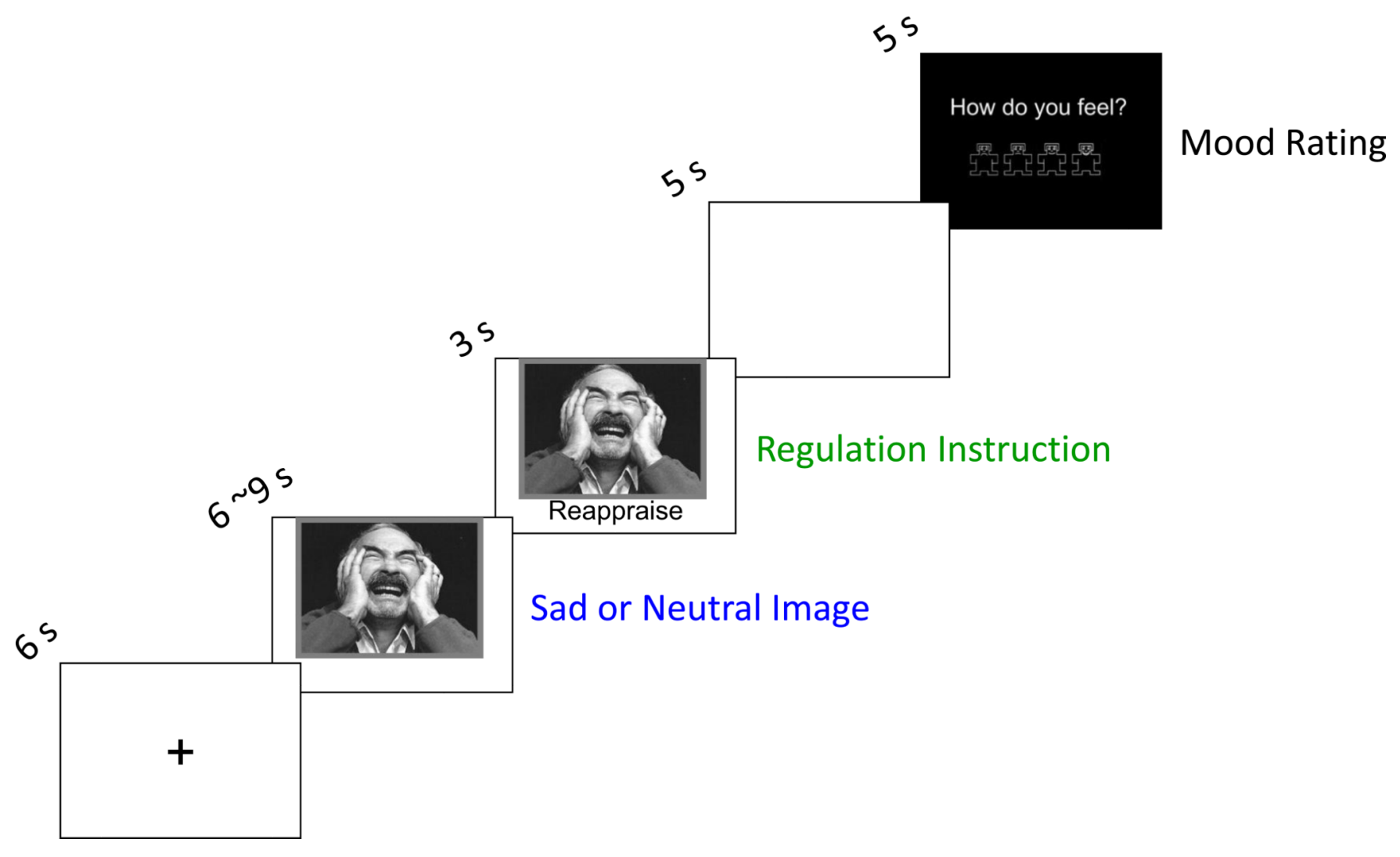

Figure 1.

Emotion regulation task. Each trial consisted of a fixation cross, a neutral or sad image, a regulation cue, a delay, and a query for current affect. 


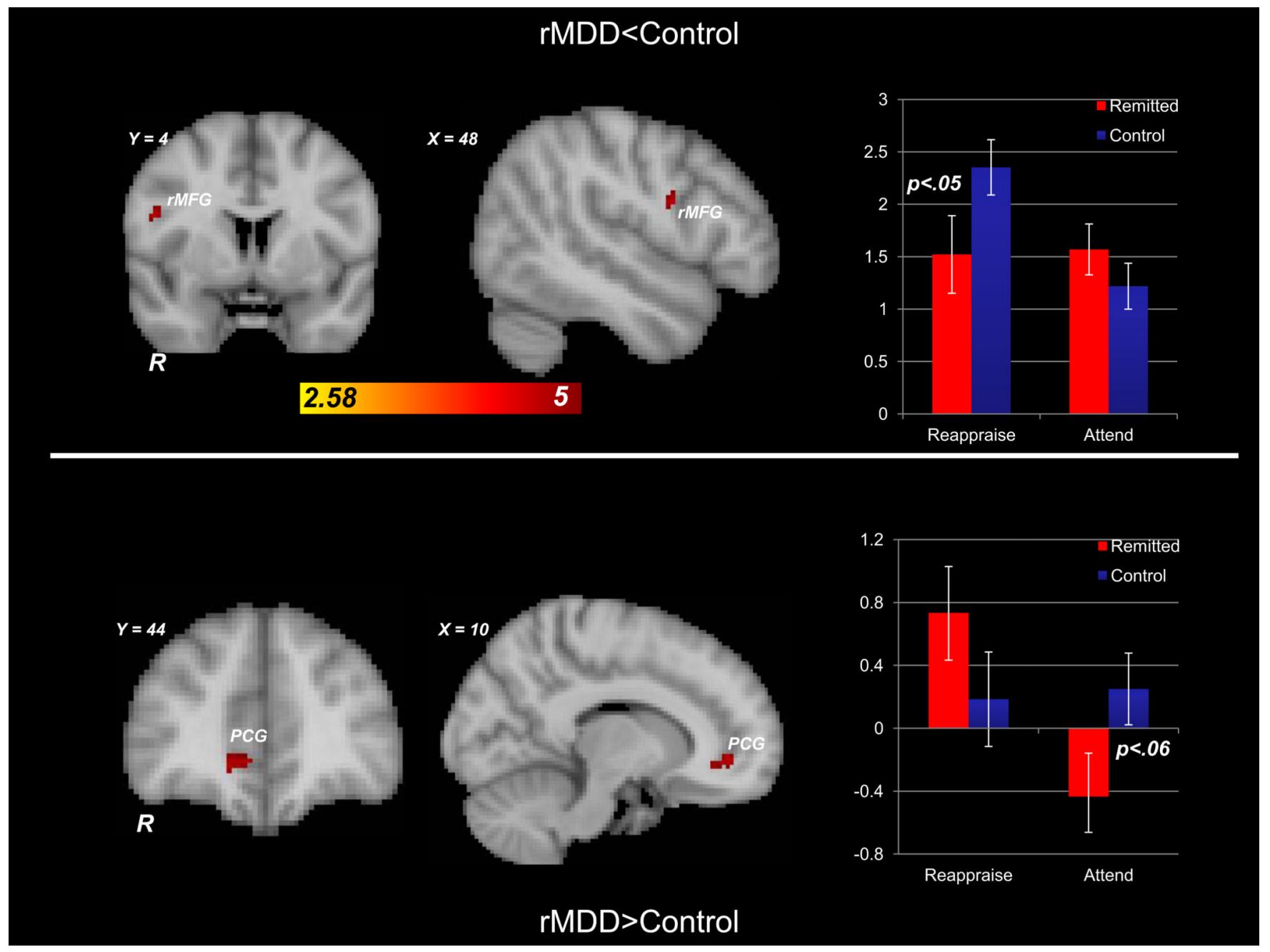

Figure 2.

The rMDD group demonstrated relatively less activation in right midfrontal gyrus (rMFG; Brodmann area 6) (top) and relatively greater activation in right paracingulate gyrus (PCG; Brodmann area 32; bottom) during reappraisal of sad images contrasted with attending to sad images. Whole-brain analyses are on the left and bar graphs from corresponding groupand condition-averaged signal intensities for the rMFG (top) and PCG (bottom) clusters are on the right. See text for results of all between- and within-groups t-tests on extracted signal intensities. 


\section{Table 1}

Demographic and symptom severity information for control and rMDD participants. Two-tailed $p$-values for between-group $t$ tests or chi-squared analyses are in the final column.

\begin{tabular}{|l|c|c|c|}
\hline & $\begin{array}{c}\text { Remitted Subjects, } \mathbf{n}=\mathbf{1 8} \\
\text { Mean (SD) }\end{array}$ & $\begin{array}{c}\text { Control Subjects, } \mathbf{n = 1 9} \\
\text { Mean (SD) }\end{array}$ & $p$-value \\
\hline Age & $24.8(4.7)$ & $27.9(6.3)$ & .10 \\
\hline Gender Male/Female & $4 / 14$ & $7 / 12$ & .33 \\
\hline Race & & & .37 \\
\hline African American & $6 \%$ & $16 \%$ & \\
\hline Caucasian & $72 \%$ & $79 \%$ & \\
\hline Asian & $11 \%$ & $5 \%$ & \\
\hline American Indian & $6 \%$ & $0 \%$ & \\
\hline Hispanic Ethnicity & $22 \%$ & $6 \%$ & .18 \\
\hline NAART VIQ & $110.7(3.3)$ & $110.2(5.1)$ & .70 \\
\hline BDI & $2.9(5.0)$ & $1.4(2.4)$ & .24 \\
\hline \# of previous depressive episodes & $1.6(0.9)$ & - & - \\
\hline \# of months since previous episode & $40.4(46.2)$ & - & - \\
\hline
\end{tabular}

NAART VIQ: North American Adult Reading Test (Uttl, 2002)

BDI: Beck Depression Inventory, $2^{\text {nd }}$ Edition (Beck et al., 1996) 


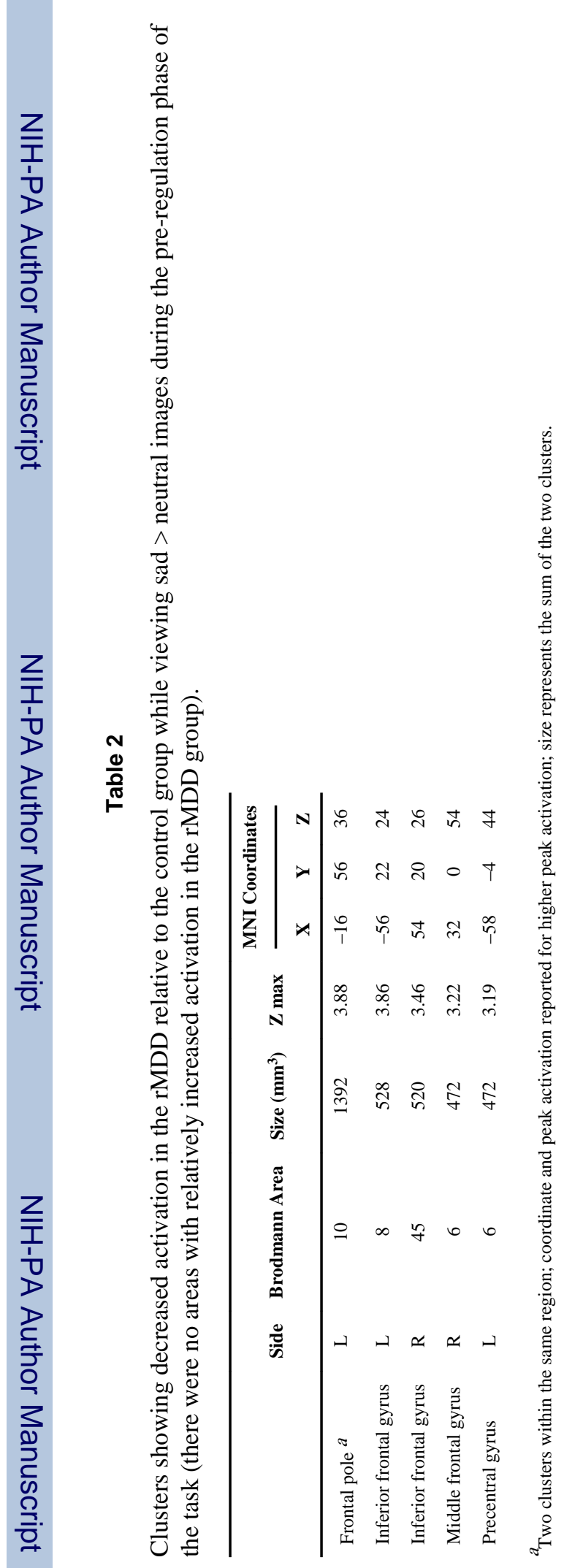




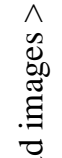

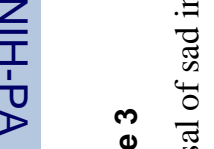

齐

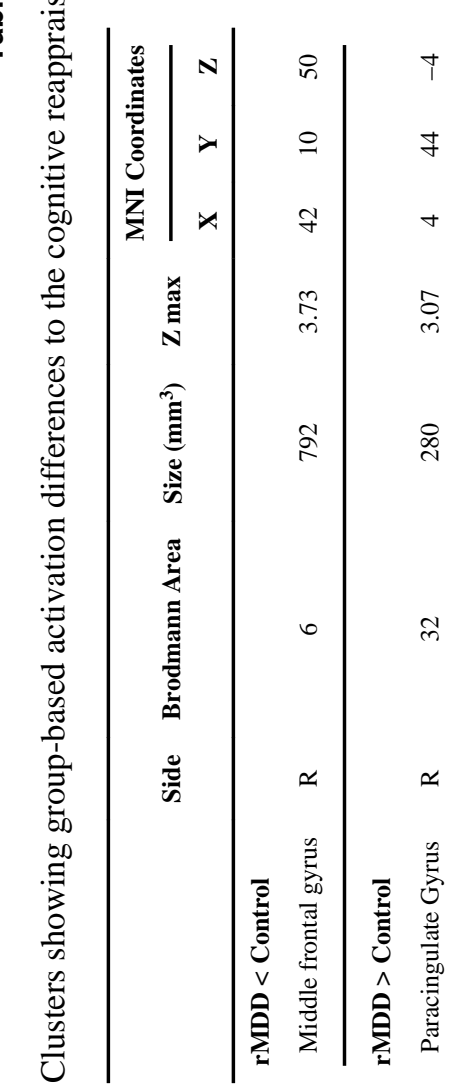

